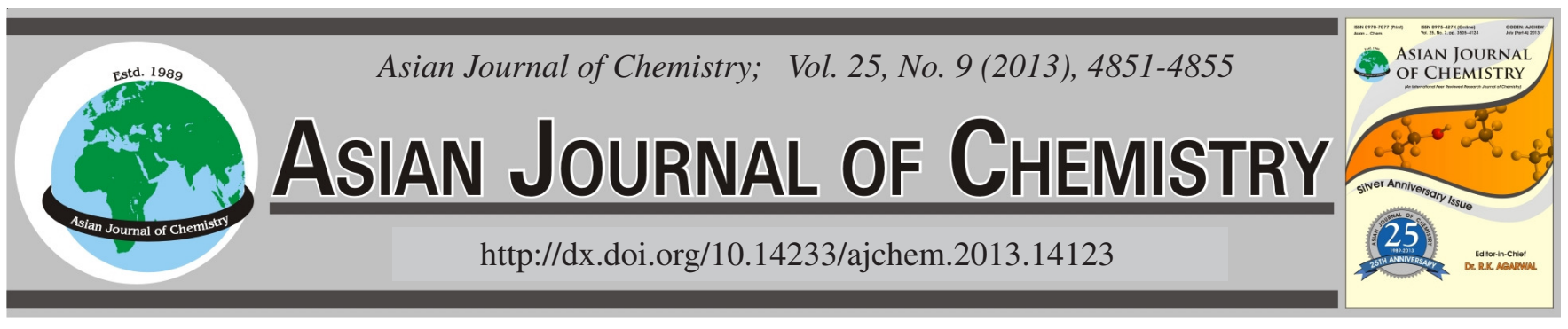

\title{
Studies of Swelling Kinetics of Carboxymethyl Cellulose-g-PMAAm-co-PNIPAm Superabsorbent Hydrogels
}

Fatemeh Soleimani $^{1, *}$, Hossein Sadeghi $^{2}$, Hadis Shahsavari $^{2}$, Arezou Soleimani $^{1}$ and Fatemeh SadeGhi ${ }^{2}$

${ }^{1}$ Young Researcher Club, Khorramabad Branch, Islamic Azad University, Khorramabad, Iran

${ }^{2}$ Department of Chemistry, Science Faculty, Khorramabad Branch, Islamic Azad University, Khorramabad, Iran

*Corresponding author: E-mail: fatisoleymani@yahoo.com

(Received: 27 May 2012;

Accepted: 8 March 2013)

AJC-13088

In this paper, we have focused on study of swelling kinetics and salt-sensitivity behaviour of a superabsorbing hydrogel based on
carboxymethylcellulose and $\mathrm{N}$-isopropylacrylamide and methacrylamide in a homogeneous solution. The swelling kinetics of the hydrogels
with various particle sizes was investigated as well. The swelling of the hydrogel showed a second order kinetics of swelling in water. In
addition, swelling measurements of the synthesized hydrogels in various chloride salt solutions was measured. Results indicated that a
swelling-loss with an increase in the ionic strength of the salt solutions.
Key Words: Carboxymethyl cellulose, Superabsorbent, Swelling kinetics, Salt-sensitivity.

\section{INTRODUCTION}

In recent years, increasing interest in natural-based superabsorbent hydrogel has developed mainly due to high hydrophilicity, biocompatibility, non-toxicity and biodegradability of biopolymers. These materials are defined as crosslinked macromolecular networks that can absorb water or physical fluids up to many times of their own weight in a short time, but are not dissolved when brought into contact with water ${ }^{1}$. Because of excellent characteristics, superabsorbent hydrogels are widely used in many fields, such as agricultural and horticultural, disposable diapers, feminine napkins, pharmaceuticals and medical applications ${ }^{2-4}$. Hence, synthesis and investigation of specific and new superabsorbent hydrogels with high absorbency, mechanical strength and initial absorption rate, has been the goal of several research groups in the past decades ${ }^{5-8}$.

Because of their exceptional properties, i.e., biocompatibility, biodegradability, renewability and non-toxicity, polysaccharides are the main part of the natural-based superabsorbent hydrogels. Carboxymethylcellulose (CMC), an anionic watersoluble polysaccharide, is important modified cellulose which is used in various fields such as detergent, food, paper and textile industries.

To the best of our knowledge, there is no published report on the synthesis of a superabsorbing hydrogel via crosslinking graft copolymerization of $\mathrm{N}$-isopropylacrylamide (NIPAAm) and methacrylamide (MAAm) onto carboxymethylcellulose
(CMC) backbones in a homogeneous solution and investigation of swelling behaviours. Hence, the objectives of this study was investigate swelling kinetics of a superabsorbent hydrogel made of CMC, NIPAAm and MAAm.

\section{EXPERIMENTAL}

The polysaccharide, carboxymethylcellulose (CMC, degree of substitution 0.52, from Condinson Co., Denmark) was of analytical grade and was used as received. N-Isopropylacrylamide (NIPAM, Merck) and methacrylamide (MAAm, Merck) were used after recrystallization. Amounium persulfate (APS, Merck) was used without purification. Methylenebisacrylamide (MBA, Fluka), was used as recieved. All other chemicals were of analytical grade. Double distilled water was used for the hydrogel preparation and swelling measurements.

Preparation of hydrogel: CMC solution was prepared in a $1 \mathrm{~L}$ reactor equipped with mechanical stirrer and gas inlet. $\mathrm{CMC}$ was dissolved in degassed distillated water. In general, $0.50 \mathrm{~g}$ of CMC was dissolved in $30.0 \mathrm{~mL}$ of distillated degassed water. The reactor was placed in a water bath preset at $60^{\circ} \mathrm{C}$. Then $0.10 \mathrm{~g}$ of ammonium persulfate (dissolved in $5 \mathrm{~mL}$ water) as an initiator was added to CMC solution and was allowed to stir for $10 \mathrm{~min}$ at $60{ }^{\circ} \mathrm{C}$. After adding initiature, variable amounts of MAAm and NIPAAm were added to the CMC solution. Methylenebisacrylamide as a crosslinker $(0.050 \mathrm{~g}$ in $5 \mathrm{~mL}$ water) was added to the reaction mixture after the 
addition of monomer and the mixture was continuously stirred for $1 \mathrm{~h}$ under argon. The total volume of reaction was $40 \mathrm{~mL}$. After $1 \mathrm{~h}$, the reaction product was allowed to cool to ambient temperature and methanol $(500 \mathrm{~mL})$ was added to the gelled product. After complete dewatering for $24 \mathrm{~h}$, the product was filtered, washed with fresh methanol $(2 \mathrm{~mL} \times 50 \mathrm{~mL})$ and dried at $50{ }^{\circ} \mathrm{C}^{8,9}$.

Instrumental analysis: Fourier transform infrared (FTIR) spectroscopy absorption spectra of samples were taken in $\mathrm{KBr}$ pellets, using an ABB Bomem MB-100 FTIR spectrophotometer (Quebec, Canada), at room temperature. The surface morphology of the gel was examined using scanning electron microscopy (SEM). After Soxhlet extraction with methanol for $24 \mathrm{~h}$ and drying in an oven, superabsorbent powder was coated with a thin layer of gold and imaged in a SEM instrument (Leo, 1455 VP).

Swelling measurements: A CMC-g-PMAAm-coPNIPAAm sample $(0.10 \mathrm{~g})$ was put into a weighed teabag and immersed in $100 \mathrm{~mL}$ distilled water or salinity solution $(0.9 \%$ wt $\mathrm{NaCl}$ ) and allowed to soak for $2 \mathrm{~h}$ at room temperature. The equilibrated swollen gel was allowed to drain by removing the teabag from water and hanging until no drop drained ( $c a$. $0.5 \mathrm{~h}$ ). The bag was then weighed to determine the weight of the swollen gel. The absorbency (equilibrium swelling) was calculated using the following equation ${ }^{4}$ :

$$
\text { Absorbency }=\frac{\left(\mathrm{W}_{\mathrm{s}}-\mathrm{W}_{\mathrm{d}}\right)}{\mathrm{W}_{\mathrm{d}}}
$$

where $\mathrm{W}_{\mathrm{s}}$ and $\mathrm{W}_{\mathrm{d}}$ are the weights of the swollen gel and the dry sample, respectively. So, absorbency was calculated as grams of water per gram of copolymer $(\mathrm{g} / \mathrm{g})$. The accuracy of the measurements was $\pm 3 \%$.

\section{RESULTS AND DISCUSSION}

Synthesis and characterization: PMAAc and PNIPAAm monomers was simultaneously grafted onto CMC in a homogenous medium using APS as a radical initiator and MBA as a crosslinking agent under an inert atmosphere.

In the first step, the persulfate initiator is decomposed under heating to generate sulfate anion-radical. The radical abstracts hydrogen from the hydroxyl group of the polysaccharide substrate to form alkoxy radicals on the substrate. So, this persulfate-saccharide redox system is resulted in active centers on the substrate to radically initiate polymerization of PMAAc and PNIPAAm monomers onto CMC backbones led to a graft copolymer. Since a crosslinking agent, e.g., MBA, is presented in the system, the copolymer comprises a crosslinked structure. The superabsorbency of this hydrogel in distilled water and salinity solution $((0.9 \% \mathrm{wt} \mathrm{NaCl})$ were investigated $^{10}$.

For identification of the hydrogel, infrared spectroscopy was used. Fig. 1 shows the IR spectroscopy of CMC-gPMAAm-co-PNIPAAm hydrogel. The superabsorbent hydrogel product comprises a CMC backbone with side chains that carry carboxamide functional groups that are evidenced by peaks at $1678 \mathrm{~cm}^{-1}$. In fact, In the spectrum of the hydrogel (Fig. 1b), new peaks are appeared at 3202 and $2937 \mathrm{~cm}^{-1}$ that may be attributed to amide $\mathrm{NH}$ stretching, asymmetric and symmetric amide $\mathrm{NH}$ bending, respectively ${ }^{9}$.

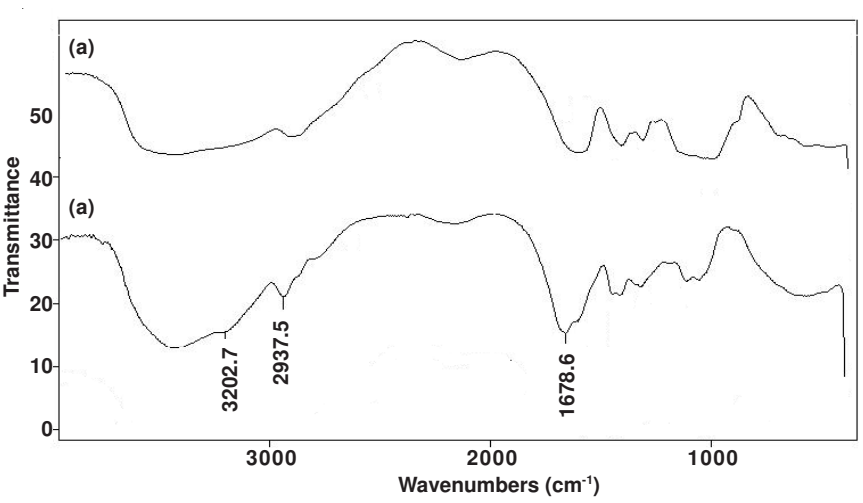

Fig. 1. FTIR spectra of (a) pure CMC and (b) CMC-g-PMAAm-coPNIPAAm hydrogel

Effect of salinity on swelling: Carboxymethyl cellulose are aggregated with various cations. Several papers have reported the interaction of carboxymethyl cellulose with cations such as $\mathrm{K}^{+}, \mathrm{Na}^{+}$and $\mathrm{Ca}^{2+}$ to form ionically crosslinked helical molecules. Degree of aggregation is strongly dependent on the type and concentration of salt added to the carboxymethylcellulose solution. However, the modified CMC prepared in the present work exhibit different behaviours because a large of crosslinked polymer chains are grafted on the polysaccharide backbone ${ }^{11}$.

To study various saline effects on the swelling behaviour of the synthesized CMC-based hydrogels, equilibrium swelling was measured in $0.15 \mathrm{M}$ solutions of $\mathrm{NaCl}, \mathrm{CaCl}_{2}$ and $\mathrm{AlCl}_{3}$ (Fig. 2). In addition, a dimensionless salt sensitivity factor (f), was defined as eqn. 2 .

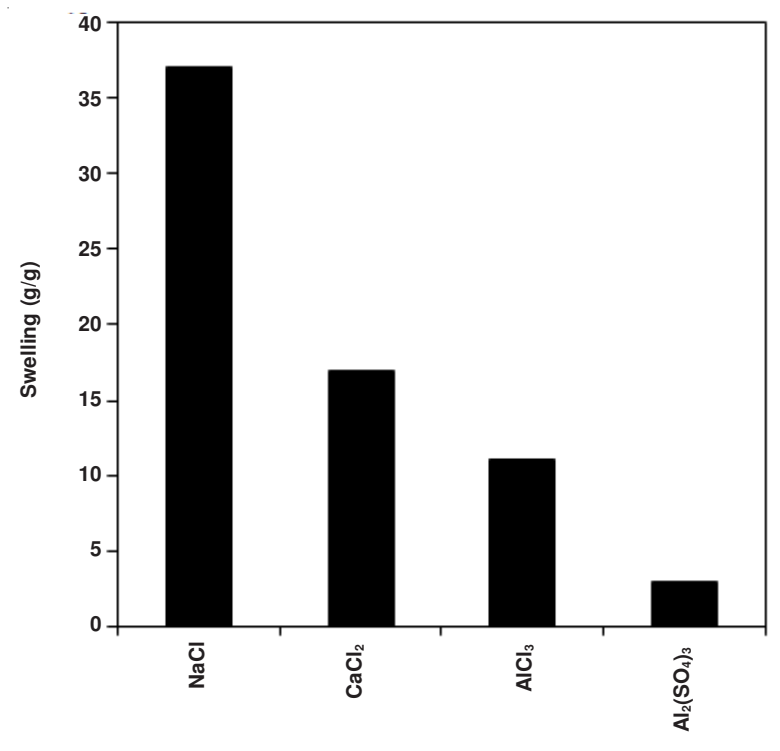

Salt solutions $(0.15 \mathrm{M})$

Fig. 2. Effect of the ionic strength of salt solutions on the swelling capacity of the CMC-g-PMAAm-co-PNIPAAm hydrogel

$$
\mathrm{f}=1-\frac{\mathrm{s}_{\mathrm{s}}}{\mathrm{s}_{\mathrm{w}}}
$$

where $S_{\mathrm{s}}$ and $\mathrm{S}_{\mathrm{w}}$ are swelling in desired salt solution and in distilled water, respectively. The f values are also given in Table-1. 


\begin{tabular}{|c|c|c|c|c|}
\hline \multicolumn{5}{|c|}{$\begin{array}{l}\text { SWELLING AND SALT SENSITIVITY OF } \\
\text { CMC-BASED HYDROGELS IN WATER AND } \\
\text { DIFFERENT SALT SOLUTIONS }(0.15 \mathrm{M})\end{array}$} \\
\hline \multirow[t]{2}{*}{$\begin{array}{l}\text { Swelling } \\
\text { medium }\end{array}$} & \multicolumn{2}{|c|}{$\begin{array}{l}\text { Before hydrolysis } \\
\text { (CMC-g-PMAAm-co- } \\
\text { PNIPAAm) }\end{array}$} & \multicolumn{2}{|c|}{$\begin{array}{l}\text { After hydrolysis (H- } \\
\text { CMC-g-PMAAm-co- } \\
\text { PNIPAAm) }\end{array}$} \\
\hline & ES $(\mathrm{g} / \mathrm{g})$ & $\mathrm{f}$ & $\mathrm{ES}(\mathrm{g} / \mathrm{g})$ & $\mathrm{f}$ \\
\hline $\mathrm{H}_{2} \mathrm{O}$ & 186 & - & 263 & - \\
\hline $\mathrm{NaCl}$ & 37 & 0.21 & 58 & 0.29 \\
\hline $\mathrm{CaCl}_{2}$ & 17 & 0.43 & 21 & 0.53 \\
\hline $\mathrm{AlCl}_{3}$ & 11 & 0.66 & 9 & 0.78 \\
\hline
\end{tabular}

In general, all swelling values in saline media are expectedly decreased. The reason is usually attributed to the reduction of osmotic pressure between the gel and the aqueous phase. Osmotic pressure, the driving force for swelling, is originated from anion-anion repulsion. In the presence of excess cations, they can shield the anions and prevent efficient electrostatic repulsion. This phenomenon is often referred to as charge "screening effect". An additional reason is increasing electrostatic attraction between anionic sites of chains and multi-valent cations leading to increased ionic crosslinking degree and consequent loss of swelling. However, the swelling in $\mathrm{NaCl}$ (wt $0.9 \%$ ) is yet considerable ( $37 \mathrm{~g} / \mathrm{g}$ compare to 186 $\mathrm{g} / \mathrm{g}$ ) for CMC- $g$-PMAAm-co-PNIPAAm. The reason for in this antisalt behaviour can be explained as follows: CMC parts of the hydrogels contain lots of carboxylic groups that can be dissociated in aqueous media more readily than the carboxamide groups of the synthetic part of H-CMC-PMAAmco-PNIPAAm. Since the carboxylate ions do not keep cations in their vicinity, the "charge screening effect" is not so effective. Thus, the resulting swelling loss is not appreciable. The most clear case indicating this fact is CMC-g-PMAAm-coPNIPAAm swelling amount in saline. It shows the lowest salt sensitivity $(\mathrm{f}=0.21)$.

A similar conclusion is recently reported by $\mathrm{Fu}$ et al. ${ }^{12}$ achieved considerable water absorbency in the case of a sulfated carboxymethylcellulose hydrogel. Therefore, the reason for high water and saline absorbency in our CMC-based hydrogels certainly a result of the presence of carboxylate groups in its CMC parts. For confirming this point, we compared swelling and salt-sensitivity for the hydrogels before and after alkaline hydrolysis (Table-1). Before the alkaline hydrolysis, carboxylic groups are the only anions assisted the hydrogel (CMC-g-PMAAm-co-PNIPAAm) to swell, so the obvious low salt-sensitivity of the superabsorbents ( $f=0.21-0.66)$ is certainly due to the presence of these anionic species. After alkaline hydrolysis, lots of carboxylate anions are added to the hydrogel systems. This superabsorbent (H-CMC-g-PMAAm-co-PNIPAAm) exhibits very high saltsensitivity (f 0.29-0.78) due to charge screening effect and, in the case of multi-valent cations, due to enhancement of ionic crosslink density.

The results shown in Fig. 3 indicate that the absorbency for the H-CMC-g-PMAAm-co-PNIPAAm hydrogels in various salt solutions also decreased with the increasing ionic strength of the salt solution. The effect of the ionic strength on water absorbency has been determined using the relation suggested by Hermans:

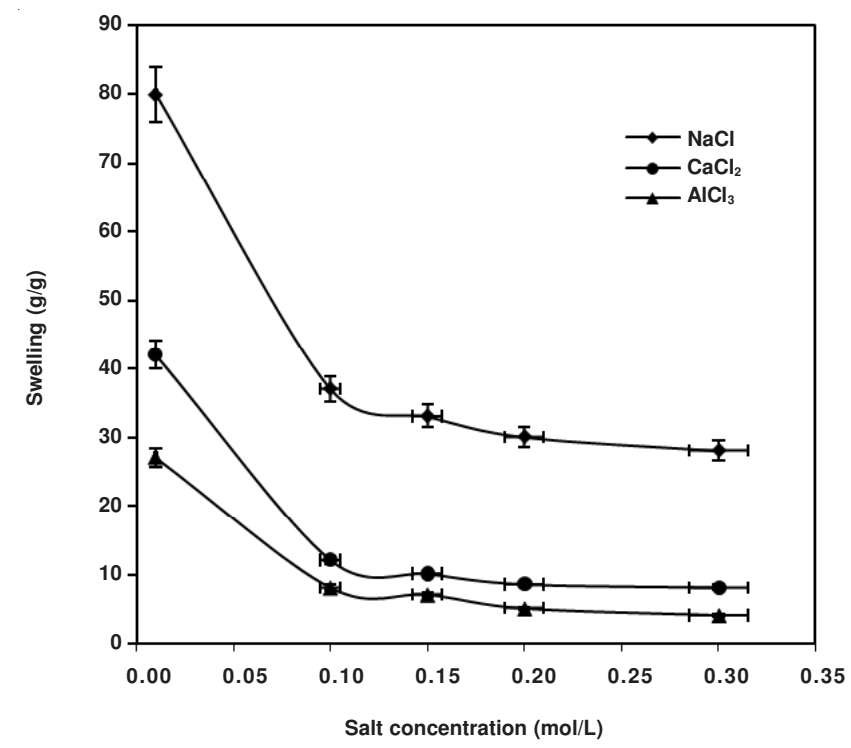

Fig. 3. Swelling capacity variation of the CMC-g-PMAAm-co-PNIPAAm superabsorbent in saline solutions with various concentrations

$$
\mathrm{Q}_{(\mathrm{eq})}^{5 / 3}=\mathrm{A}+\frac{\mathrm{Bi}^{2}}{\mathrm{I}}
$$

which $\mathrm{Q}_{(\mathrm{eq})}$ is the equilibrium water absorbency, $\mathrm{I}$ is the ionic strength of the external solution and A and B are the empirical parameters. At low ionic strengths, the concentration of bond charges within the hydrogel network exceeds the concentration of salt in the external solutions, a large ion-swelling pressure causes the hydrogel to expand, thereby lowering the concentration of ions within the hydrogel. As the external salt concentration rises, the difference between the internal and external ion concentration decreases and the hydrogel deswells. The hydrogel continues to deswell with increasing external salt concentration until the mobile-ion concentrations inside and outside are approximately equal. These phenomenons can also be explained on the basis of repulsion between fixed charged groups on the hydrogel. As ionic strength increases, repulsion is shielded and the hydrogel deswell (charge screening effect).

Swelling kinetics studies: In practical applications, a higher swelling rate is required as well as a higher swelling capacity. It is well known that the swelling kinetics for the absorbents is significantly influenced by factors such as swelling capacity, size distribution of powder particles, specific size area and composition of polymer. The influences of these parameters on the swelling capacity have been investigated by various workers ${ }^{8,13}$. For example, the dependency of water absorbency of superabsorbent polymers on particle size investigated by Metz et al. ${ }^{10}$. Results indicated that as the particle size became smaller, the rate of absorption increased. This may be attributed to an increase in surface area with decreasing particle size of samples. Results in Fig. 4 that shown the dynamic swelling behaviour of the superabsorbent hydrogel with various particle sizes in water, confirm this fact. According to the figure, the rate of water absorbency sharply increases and then begins to level off. For preliminary study of swelling kinetics, a "Voigt-based model" may be used for fitting the swelling data (eqn. 4$)^{13}$ : 


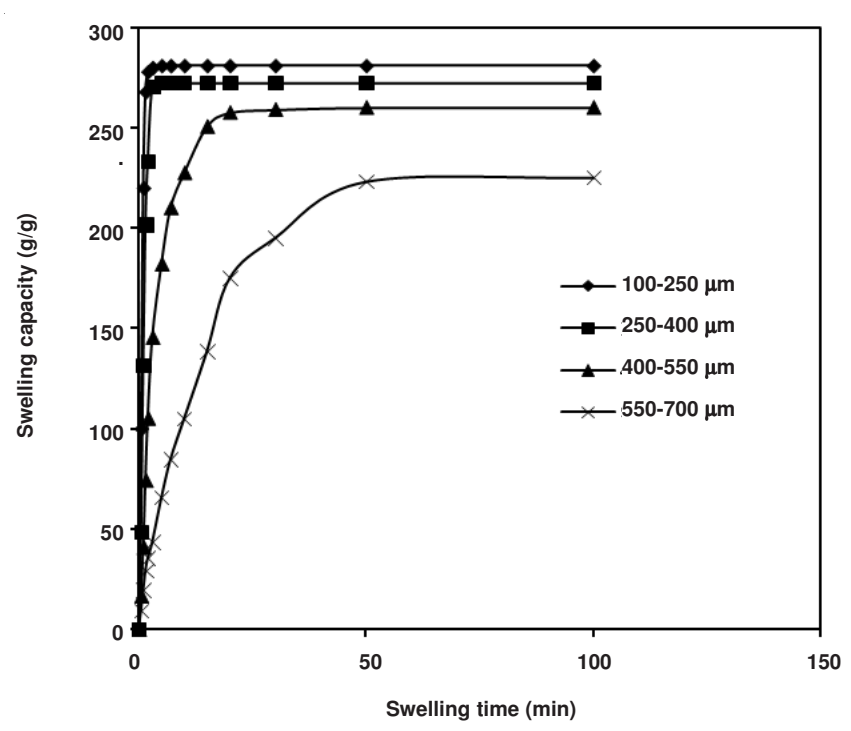

Fig. 4. Representative swelling kinetics of the CMC-g-PMAAm-coPNIPAAm superabsorbent hydrogel with various particle sizes

$$
\mathrm{S}_{\mathrm{t}}=\mathrm{S}_{\mathrm{e}}\left(1-\mathrm{e}^{-\mathrm{t} / \tau}\right)
$$

where $S_{\mathrm{t}}$ is the swelling at time $t, S_{\mathrm{e}}$ is the equilibrium swelling (power parameter) and $\tau$ is the rate parameter. The $\tau$ value is a measure of swelling rate (i.e., the lower the $\tau$ value, the higher the rate of swelling). For calculate the rate parameter, by using the above formula and a little rearrangement, one can be plot ln $\left(1-\mathrm{S}_{\mathrm{t}} / \mathrm{S}_{\mathrm{e}}\right)$ versus time $(\mathrm{t})$. The slope of the straight line fitted $($ slope $=-1 / \tau)$ gives the rate parameter. The rate parameters for superabsorbent are found to be 3.9, 7.8, 10.1 and $11.4 \mathrm{~min}$ for superabsorbent with particle sizes of 100-250, 250-400, 400-550 and 550-700 $\mu \mathrm{m}$, respectively. According to the smaller $\tau$ value, the swelling of the superabsorbent with 100$250 \mu \mathrm{m}$ particle sizes is faster than other counterparts.

We analyzed the swelling kinetics in order to find out whether swelling follows first or second order kinetics. We adopted the procedure followed by Quintana et al. For the first order kinetics, rate of swelling at any time is proportional to the water content before the equilibrium absorbed water $\left(\mathrm{W}_{\infty}\right)$ has been reached. The swelling can be expressed as eqn. $5:$

$$
\frac{\mathrm{dW}}{\mathrm{dt}}=\mathrm{K}\left(\mathrm{W}_{\infty}-\mathrm{W}\right)
$$

where $\mathrm{W}$ is the water content of the superabsorbent at time $\mathrm{t}$ and $\mathrm{K}$ is a constant.

Upon integration of eqn. 5 between the limits $t=0$ to $t$ and $\mathrm{W}=0$ to $\mathrm{W}$, the following expression can be obtained:

$$
\ln \frac{\mathrm{W}_{\infty}}{\mathrm{W}_{\infty}-\mathrm{W}}=\mathrm{Kt}
$$

If the swelling process of superabsorbent follows a first order kinetics, the plot of the variation of $\ln \left(\mathrm{W}_{\infty} / \mathrm{W}_{\infty}-\mathrm{W}\right)$ as a function of time should give a straight line. But none of the swelling studies in water followed eqn. 6 , as is clear from Fig. 5.

Considering the second order kinetics, the swelling rate at any time may be expressed as eqn. 7 :

$$
\frac{\mathrm{dW}}{\mathrm{dt}}=\mathrm{K}\left(\mathrm{W}_{\infty}-\mathrm{W}\right)^{2}
$$

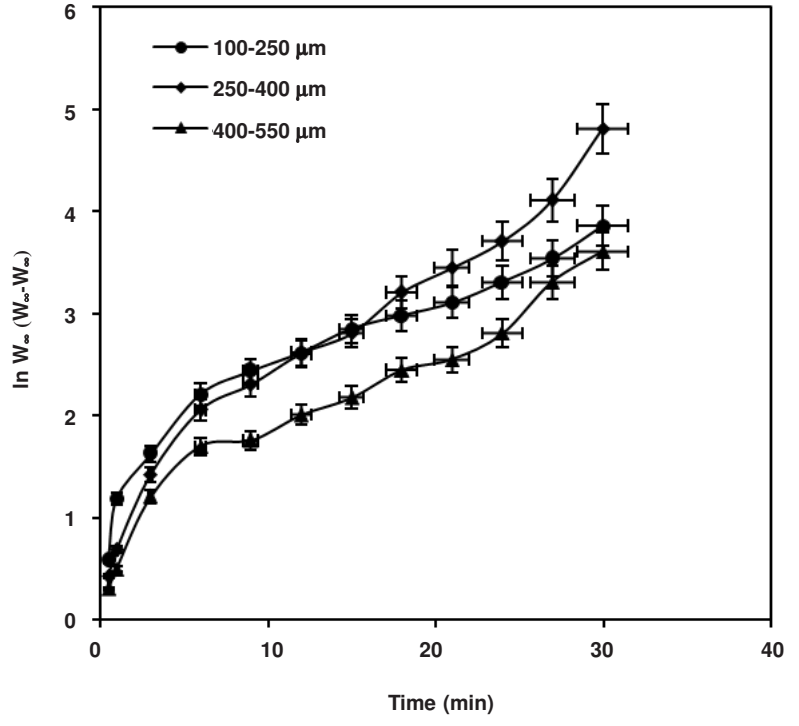

Fig. 5. Plot of $\ln \left(\mathrm{W}_{\infty} / \mathrm{W}_{\infty}-\mathrm{W}\right)$ versus time, according to eqn. 7 (first order kinetics) for the superabsorbent hydrogels with different particle sizes

Integration eqn. 7 with the limits $\mathrm{t}=0$ to $\mathrm{t}$ and $\mathrm{W}=0$ to $\mathrm{W}$ and after rearrangement, the following equation is obtained:

$$
\frac{\mathrm{t}}{\mathrm{W}}=\frac{1}{\mathrm{KW}_{\infty}^{2}}+\frac{1}{\mathrm{~W}_{\infty}} \mathrm{t}
$$

According to this equation, the swelling data must fit a straight line with a slope of $1 / \mathrm{W}_{\infty}$ and an ordinate of $1 / \mathrm{KW}_{\infty}^{2}$. The variation of $\mathrm{t} / \mathrm{W}$ against time is plotted in Fig. 6. It was found that swelling data of superabsorbent in water gives straight lines. So, the swelling of the synthesized superabsorbent composites with various particle sizes obey second order kinetics.

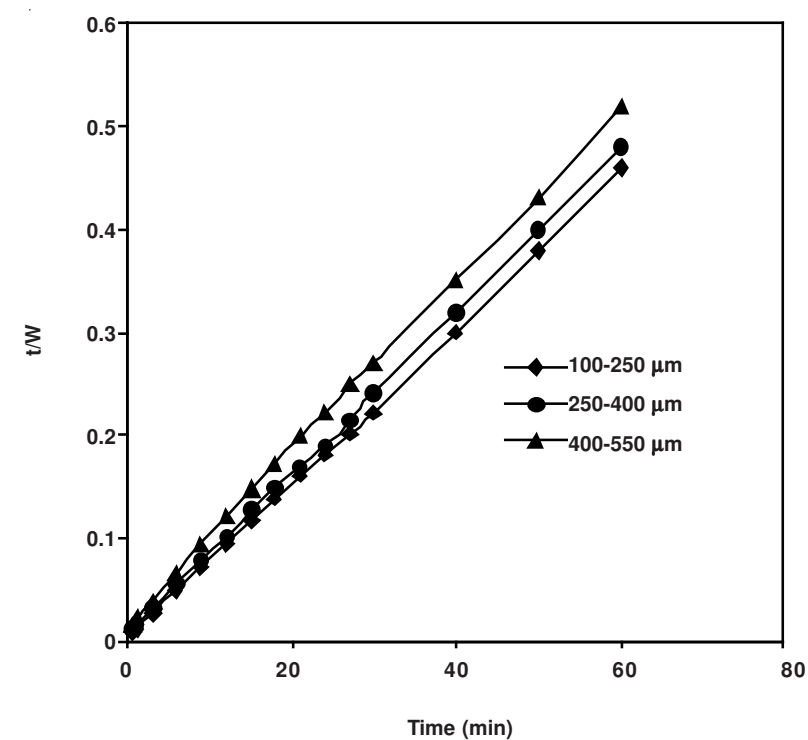

Fig. 6. Plot t/W vs. time according to eqn. 8 (second order kinetics) for superabsorbent hydrogels with various particle sizes

\section{Conclusion}

In this paper, the swelling kinetics of the synthesized hydrogels with various particle sizes was investigated. The 
swelling measurement in various salt solutions shows a swellingloss behaviour. This behaviour can be attributed to charge screening effect and ionic crosslinking for mono- and multivalent cations, respectively. The rate of water uptake is also increased with decreasing the particle size of the hydrogels. This can be attributed to high surface avability of small particle size of superabsorbing polymers when brought into contact with water. In addition, according to the dynamic rate measurements, the swelling of the hydrogel showed a second order kinetics of swelling in water.

\section{REFERENCES}

1. M.M. Ibrahim, E.M. Flefel and W.K. El-Zawawy, J. Appl. Polym. Sci., 84, 2629 (2002)

2. E.M. Flefel, M.M. Ibrahim, W.K. El-Zawawy and A.M. Ali, Polym. Adv. Technol., 13, 541 (2002).
3. A.A. Berlin and V.N. Kislenco, Prog. Polym. Sci., 17, 765 (1992).

4. A. Pourjavadi and M.J. Zohuriaan-Mehr, Starch/Starke, 54, 140 (2002).

5. C.D. Doyle, Anal. Chem., 33, 77 (1961).

6. V.D. Athawale and V. Lele, Starch/Starke, 52, 205 (2000).

7. D.W. Jenkins and S.M. Hudson, Chem. Rev., 101, 3245 (2001).

8. J.M. Joshi and S.V. Kumar, Polymer, 47, 2198 (2006).

9. B. Singh, N. Chauhan and S. Kumar, Carbohydr. Polym., 73, 446 (2008).

10. S.J. Metz, W.J.C. Van de Ven and J. Potreck, J. Membr. Sci., 251, 29 (2005).

11. G. Mino and S. Kaizerman, J. Polym. Sci., 1, 242 (1958).

12. G. Fu, J. Zhao, H. Yu, L. Liu and B. He, React. Funct. Polym., 67, 442 (2007)

13. J.H. Ye, J.J. Dong, J.L. Lu, X.Q. Zheng, J. Jin, H. Chen and Y.R. Liang, Carbohydr. Polym., 81, 441 (2010).

13. P.K. Pandey, A. Srivastava, J. Trpathy and K. Behari, Carbohydr. Polym., 65, 414 (2006). 